\title{
Clipes de titânio no tratamento de aneurismas
}

\section{saculares intracranianos}

\author{
Mario G. Siqueira*, Osmar J.S. Moraes ${ }^{\star *}$, Marco T.S. Santos ${ }^{\star \star *}$, Nelci Zanon ${ }^{\star \star *}$, \\ Aguinaldo C. Zuppani****, Marco A.M. Medeiros ${ }^{\star * * * *}$
}

Serviços de Neurocirurgia e de Radiologia do Hospital Santa Marcelina, São Paulo - SP

\begin{abstract}
RESUMO
Este trabalho descreve a utilização de 25 clipes de titânio no tratamento de 20 pacientes com hemorragia subaracnóide aneurismática. Todos os pacientes apresentavam hemorragias de graus I e II na escala de Hunt e Hess, provocadas por aneurismas da circulação anterior. O resultado do tratamento cirúrgico foi bom, não ocorrendo qualquer complicação, precoce ou tardia, que pudesse ser atribuída aos clipes de titânio. As dimensões médias dos artefatos provocados pelos clipes na tomografia computadorizada (TC) (comprimento) e ressonância magnética (RM) (volume) foram de $17,65 \mathrm{~mm}$ e $1,64 \mathrm{~cm}^{3}$, respectivamente. Os clipes de titânio demonstraram ser cirurgicamente seguros e efetivos e, quando comparados com os clipes de liga de cobalto disponíveis no comércio, produziram artefatos bem menores nas imagens obtidas pela TC e RM, devido a sua menor susceptibilidade magnética. Com base nessas importantes vantagens, acreditamos que os clipes de titânio devam ser considerados como a opção ideal para uso rotineiro em cirurgias de aneurismas.
\end{abstract}

\section{PALAVRAS-CHAVE}

Aneurisma intracraniano. Clipes de liga de cobalto. Clipes de titânio.

\section{ABSTRACT}

Titanium clips in the treatment of intracranial saccular aneurysms

This report describes the clinical use of 25 titanium clips in the treatment of 20 patients with aneurysmal subarachnoid hemorrhage. All patients had Hunt and Hess Grade I-Il hemorrhages from aneurysms of the anterior circulation. The surgical outcome was good in the entire series, with no immediate or delayed complication related to the titanium clips. The average clip artifacts on the postoperative computerized tomography (CT) (lenght) and magnetic resonance imaging (MRI) (volume) was 17.65 milimeters and 1.64 cubic centimeters, respectively. The titanium aneurysm clips seem to be surgically safe and effective and to reduce the clip artifacts on CT and MRI studies, when compared with commercially available cobalt alloy clips. Based on these important advantages over conventional clips, titanium clips should be considered as the ideal option for routine use in aneurysm surgery.

\section{KEY WORDS}

Cerebral aneurysms. Cobalt alloy clips. Titanium clips.

\section{Introdução}

A crescente utilização de estudos de imagem por ressonância magnética (RM) tem exigido que cada vez mais se utilize implantes biológicos compostos de materiais que não tenham atividade ferromagnética, tanto por razões de

\footnotetext{
* Chefe do Serviço de Neurocirurgia.

** Chefe de Clínica do Serviço de Neurocirurgia.

*** Médico Assistente do Serviço de Neurocirurgia

**** Chefe do Serviço de Radiologia.

***** Médico Assistente do Serviço de Radiologia.
} 
segurança como para minimizar os artefatos provocados pelo implante. Dentre esses, destaca-se o titânio, material implantável de uso crescente em medicina, por sua durabilidade, excelente resistência à corrosão, biocompatibilidade e compatibilidade com a RM ${ }^{6,13,16}$.

A maioria dos clipes para aneurisma disponíveis no comércio é manufaturada a partir de ligas de cobalto. Esses clipes são duráveis, biocompatíveis e sem atividade ferromagnética ${ }^{3,14,15,17}$. No entanto, embora compatíveis com a RM, esses clipes produzem artefatos que degradam a qualidade das imagens, distorcem as estruturas demonstradas e podem comprometer o potencial de diagnóstico dos estudos ${ }^{1,2,5,8,12,17}$.

Em 1966, foram publicados os primeiros estudos sobre o clipe de Spetzler ${ }^{10,11}$, produzido a partir de titânio quimicamente puro $(99,5 \%)$, inicialmente pela Elekta Instruments Inc. (EUA) e atualmente pela NMTNitinol Medical Technologies (EUA). Esses clipes encontram-se disponíveis no mercado com 55 diferentes configurações de tamanho e/ou formato.

Com a finalidade de avaliar o comportamento desses clipes e compará-los com os clipes de liga de cobalto de uso corrente em nosso meio, realizamos este estudo.

\section{Pacientes e métodos}

Foi analisada uma série de 20 pacientes com hemorragia subaracnóide aneurismática internados no Serviço de Neurocirurgia do Hospital Santa Marcelina, nos quais foram implantados 25 clipes manufaturados a partir de fios de titânio grau 4 (quimicamente puro), cujo conteúdo de ferro encontra-se abaixo de $0,50 \%$ em peso.

Com a finalidade de reduzir possíveis variáveis, selecionamos para o estudo pacientes em graus I e II na escala de Hunt e Hess, portadores de aneurismas relativamente simples, da circulação anterior, que foram operados mais de dez dias após a hemorragia subaracnóidea.

No pré-operatório, todos os pacientes foram submetidos a tomografia computadorizada (TC) do crânio em aparelho Tomoscan SR-4000 (Philips, Holanda) e a estudo angiográfico digital cerebral. No pós-operatório, os pacientes foram submetidos a estudo radiológico simples do crânio, TC, RM e angiografia digital cerebral. Os estudos de ressonância magnética foram realizados em aparelho Gyroscan T10-NT de 1.0 tesla (Philips, Holanda).

Para avaliar de forma quantitativa os artefatos produzidos na TC, foram selecionados os cortes em que se manifestavam com maior intensidade e suas extensões foram medidas linearmente (Fig. 1).

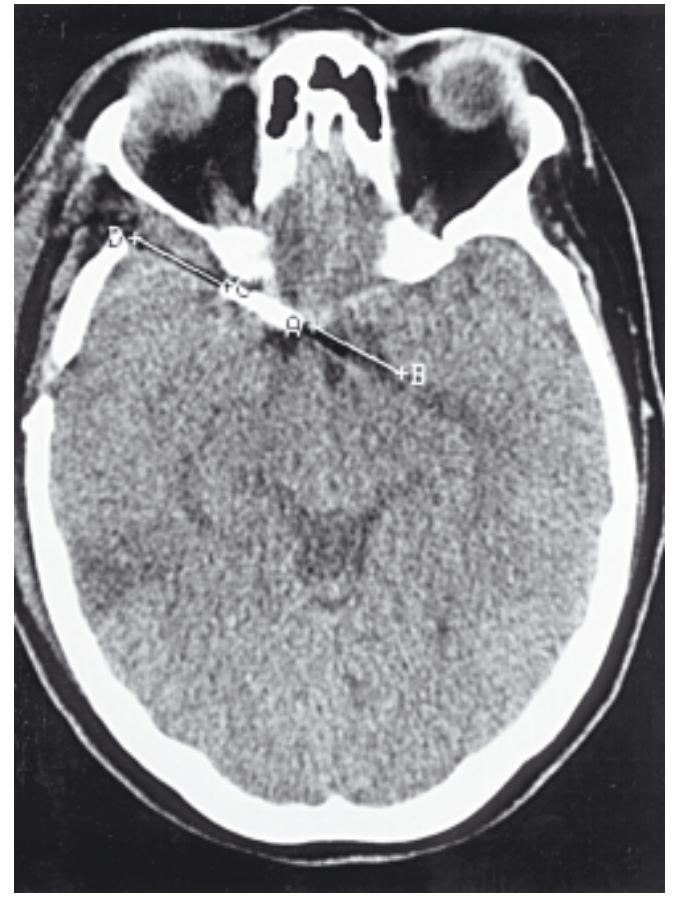

Figura 1 - Tomografia computadorizada de crânio em corte axial, sem contraste, demonstrando o método de mensuração dos artefatos provocados pelos clipes de aneurisma.

A aferição aproximada do volume do artefato na RM foi efetuada em imagens baseadas em T1, na seqüência gradiente-echo, na incidência coronal. Foram obtidos cortes finos (espessura de 2-3 mm) na área do artefato para produzir o maior número de imagens possíveis deste (de 15 a 20). Ao redor de cada imagem foi traçada uma linha contornando o artefato, que permitiu o cálculo da área de cada imagem-artefato. Uma vez obtidas todas as áreas, o computador do aparelho de RM nos forneceu cálculo volumétrico aproximado (Fig. 2).

Os artefatos provocados pelos clipes de titânio foram comparados com os encontrados na avaliação de cinco clipes de liga de cobalto.

Os pacientes foram acompanhados por um período médio de três meses.

\section{Resultados}

À exceção de uma curva na base de uma das lâminas, que as alinha e evita o "tesouramento", e de um padrão diferente de serrilhado destas (tipo DeBakey), os clipes de titânio são de formato bastante semelhante aos demais clipes disponíveis (Fig. 3). A aplicação e reajustes de posição dos clipes foram executados sem qualquer dificuldade. O manuseio intra-operatório foi 


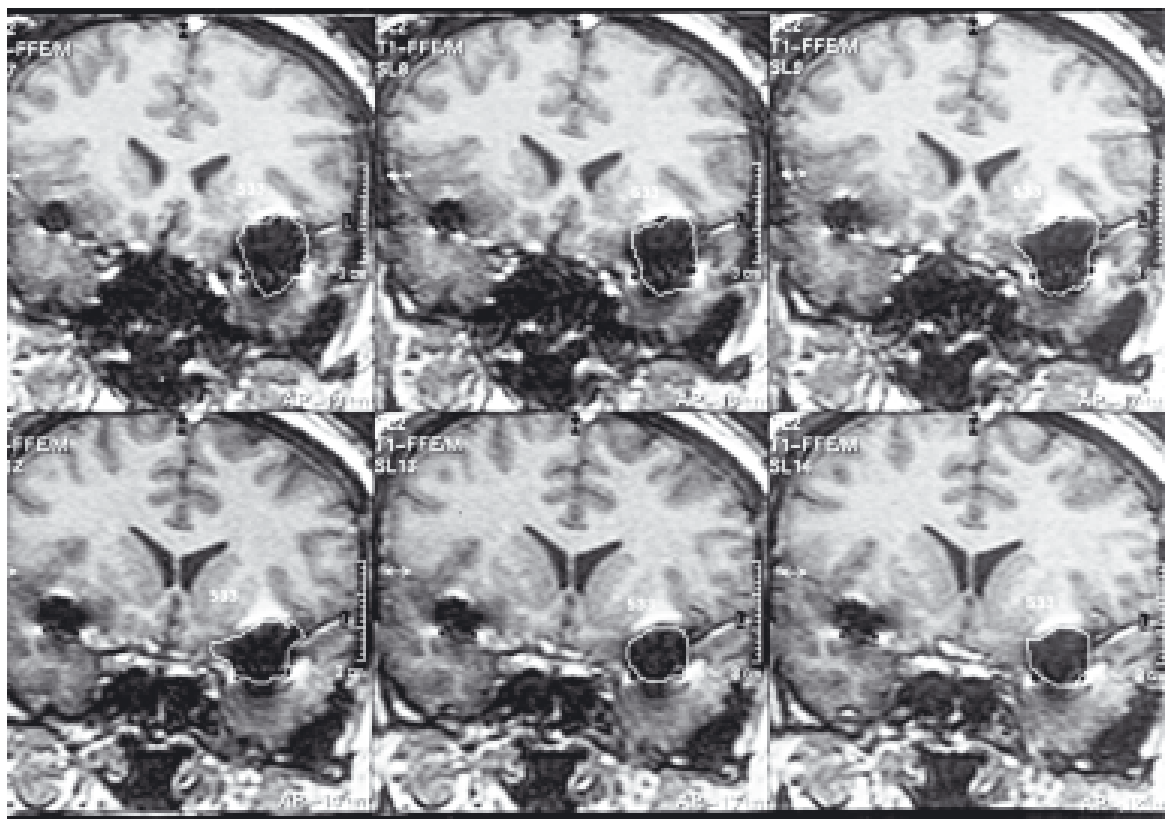

Figura 2 - Ressonância magnética do crânio em corte coronal, com imagem baseada em $T 1$, sem contraste. Delimitação das áreas de artefato provocadas pelo clipe de aneurisma, para posterior cálculo aproximado do volume.

grandemente facilitado pelo delgado perfil tanto dos clipes quanto dos aplicadores (Fig. 3) e pelo padrão de serrilhado das lâminas, que dificulta o deslizamento após a aplicação.

Os estudos por imagem pós-operatórios foram bem tolerados pelos pacientes.

A menor densidade do clipe de Spetzler é bem visível nas radiografias simples de crânio, sendo, em geral, passível de diferenciação dos demais clipes devido à citada curvatura de uma de suas lâminas (Fig. 4).

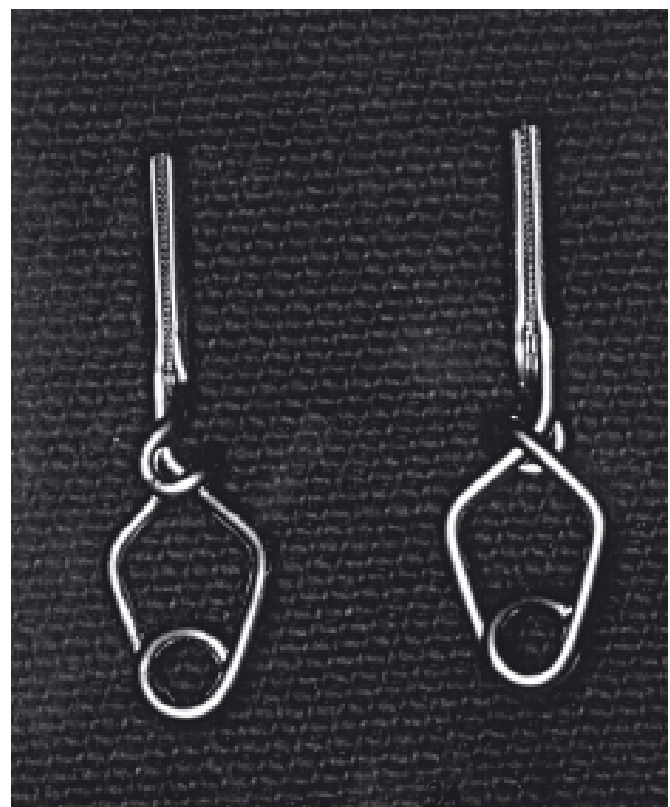

O estudo angiográfico digital pós-operatório confirmou o posicionamento adequado dos clipes, sem enchimento residual dos aneurismas tratados.

$\mathrm{Na} \mathrm{TC}$, os artefatos foram do tipo "em riscas" (Fig. 5) e na RM apresentaram-se sob a forma de "ausência de sinal", por vezes margeados parcialmente por um fino halo de hiperdensidade (Fig. 6).

Os efeitos-artefato médios provocados pelos clipes de titânio foram de 17,65 mm lineares na TC e de $1,64 \mathrm{~cm}^{3}$ na RM. Já os artefatos médios pro-

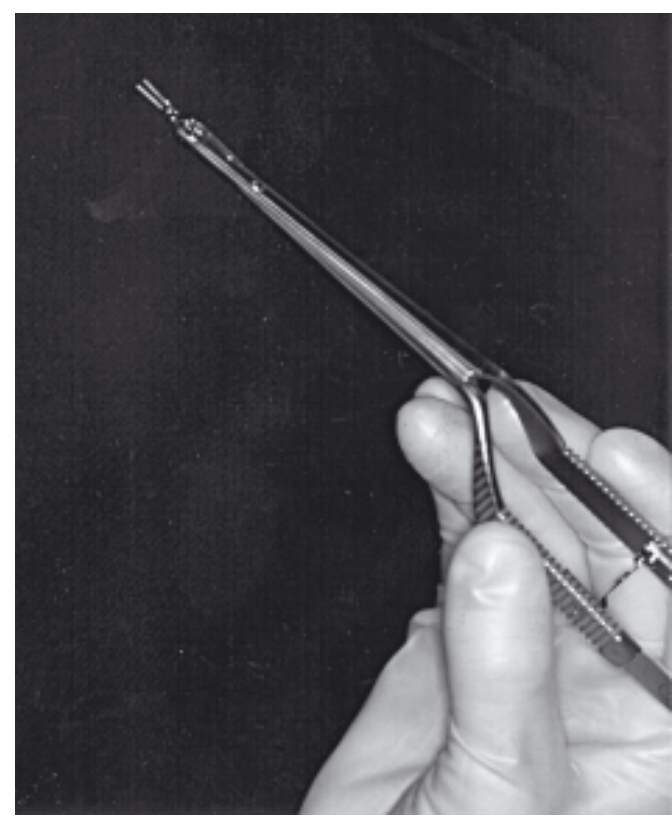

Figura 3 - Clipes de titânio e aplicador. Esquerda: exemplo de dois clipes retos. Observe a curva na base de uma das lâminas. Direita: clipe montado no aplicador. Observe o perfil delgado de ambos. 


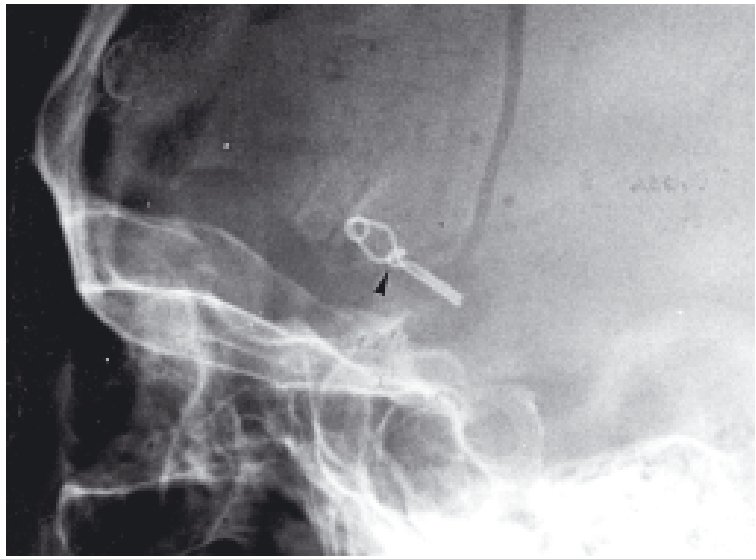

Figura 4 - Radiografia simples de crânio em perfil demonstrando clipe de aneurisma de titânio. Identificação facilitada pela curvatura de uma de suas lâminas (seta).

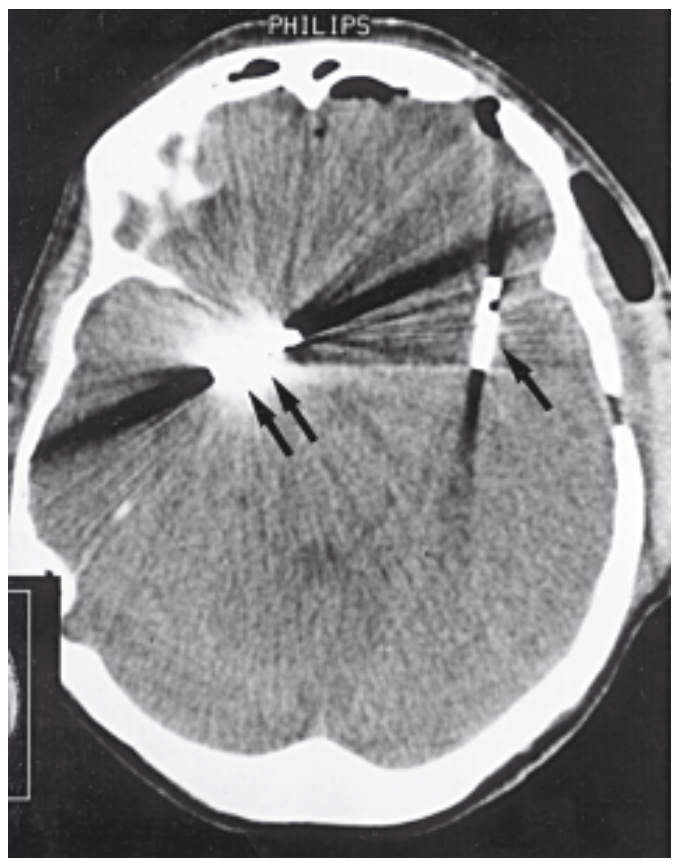

Figura 5 - Tomografia computadorizada de crânio em corte axial, sem contraste, demonstrando os artefatos em riscas relacionados aos clipes de aneurisma. Note a menor intensidade dos artefatos provocados pelo clipe de titânio (seta), quando comparados com aqueles provocados pelo clipe de liga de cobalto (seta dupla).

vocados pelos clipes de liga de cobalto foram de $49,06 \mathrm{~mm}$ na TC e de $3,81 \mathrm{~cm}^{3}$ na RM. Isso significa que os artefatos produzidos pelos clipes de liga de cobalto são 2,7 vezes maiores na TC e 2,3 vezes maiores na RM, que aqueles provocados pelos clipes de titânio.

Durante o período de internação para tratamento dos aneurismas, bem como durante o acompanhamento ambulatorial, não foi evidenciado qualquer tipo de complicação que pudesse ser diretamente atribuído aos clipes de titânio.

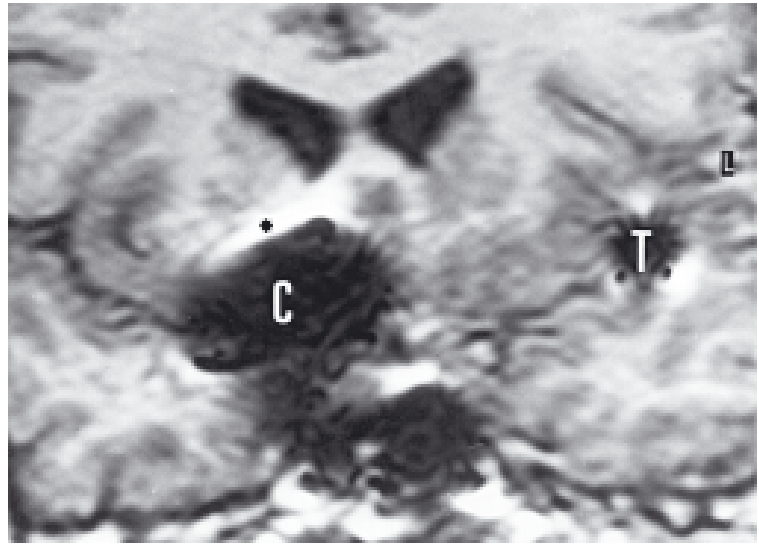

Figura 6 - Ressonância magnética do crânio em corte coronal, com imagem baseada em T1, sem contraste. Artefatos sob a forma de ausência de sinal, parcialmente margeados por halo de hiperdensidade (*). Note a diferença da intensidade dos artefatos provocados pelo clipe de titânio $(T)$ e pelo clipe de liga de cobalto $(C)$.

\section{Discussão}

De uma maneira ideal, os clipes de aneurismas devem satisfazer três critérios ${ }^{11}$ : (a) devem gerar forças de fechamento suficientes; (b) devem ser manufaturados a partir de um material durável e que o tecido cerebral tolere bem; (c) não podem ser afetados pelos campos magnéticos dos equipamentos de RM.

Os clipes para tratamento de aneurismas cerebrais eram, inicialmente, feitos de aço inoxidável. Embora fortes e resistentes à corrosão, a estrutura desses clipes tornava-os ferromagnéticos, o que, além de produzir grandes artefatos na TC e na RM, impedia a realização de estudos por RM pelo risco de deslocamento9.

Os clipes de Yasargil (Aesculap, Alemanha) e de Sugita (Mizuho, Japão), ambos importados, juntamente com os clipes nacionais da ADCA (Belo Horizonte) são os mais disponíveis atualmente no mercado e, portanto, os mais utilizados pelos neurocirurgiões brasileiros. Todos esses clipes são produzidos a partir de uma liga composta principalmente de cobalto e cromo.

Segundo afirmam os fabricantes, os clipes de liga de cobalto saem das fábricas sem atividade ferromagnética. No entanto, foi recentemente demonstrado que um pequeno percentual desses implantes pode ser "contaminado" no manuseio, armazenagem ou reesterilização após a distribuição, adquirindo discretas propriedades ferromagnéticas? ${ }^{7}$.̀̀ medida que começam a ser utilizados equipamentos de ressonância magnética de maior potência (até 3.0 tesla) ${ }^{4}$, essas constatações passam a ser preocupantes pelo alto risco de interação entre os clipes e o campo magnético.

Embora tanto as ligas de cobalto como o titânio sejam materiais sem propriedades ferromagnéticas, 
suas susceptibilidades magnéticas diferem ${ }^{10}$, sendo maior nos clipes de cobalto, que incluem na composição de suas ligas pequenos teores de metais magnéticos, como ferro e níquel.

As características mecânicas (pressões de abertura e fechamento) dos clipes de titânio, assim como suas características de biocompatibilidade, de performance e de segurança, são comparáveis às apresentadas pelos clipes de liga de cobalto ${ }^{11}$. No entanto, quando analisadas as características de imagem e os aspectos de segurança na realização de estudos por ressonância magnética, conclui-se que os clipes de titânio representam um real avanço tecnológico que deveria posicioná-los como a primeira opção quando da escolha de clipes para o tratamento cirúrgico dos aneurismas cerebrais.

\section{Conclusão}

Os riscos em potencial e a degradação da imagem associados à exposição de clipes de liga de cobalto a ambientes de ressonância magnética levaram à procura de materiais com baixa susceptibilidade ferromagnética. O titânio demonstrou ser o material disponível ideal. Os testes mecânicos e de biocompatibilidade efetuados em clipes de titânio, assim como seu desempenho na utilização clínica, apresentaram resultados semelhantes aos aplicados aos clipes de liga de cobalto. No entanto, no confronto dos estudos por imagem, os clipes de titânio demonstraram ser bem superiores. Essa vantagem, associada à maior segurança durante exposição à ressonância magnética, coloca-os em posição de primeira escolha para o tratamento cirúrgico de aneurismas cerebrais.

\section{Referências:}

1. BARRAFATO D, HENKELMAN RM: Magnetic resonance imaging and surgical clips. Can J Surg 27:509-512, 1984.

2. BECKER RL, NORFRAY JF, TEITELBAUM GP, BRADLEY WG Jr, JACOBS JB, WACASER L, RIEMAN $R L$ : MR imaging in patients with intracranial aneurysm clips. AJNR Am J Neuroradiol 9:885-889, 1988.

3. DUJOVNY M, KOSSOVSKY N, KOSSOVSKY R, VALDIVIAR, SUK JS, DIAZ FG, BERMAN SK, CLEARY $W$ : Aneurysm clip motion during magnetic resonance imaging: In vivo experimental study with metallurgic factor analysis. Neurosurgery 17:543-548, 1985.

4. FUJIIY, NAKAYAMA N, NAKADA T: High-resolution T2reversed magnetic resonance imaging on a high magnetic field system. J Neurosurg 89:492-495, 1988.
5. GO KG, KAMMAN RL, MOOYAART EL: Interaction of metallic neurosurgical implants with magnetic resonance imaging at 1.5 tesla as a cause of image distortion and of hazardous movement of the implant. Clin Neurol Neurosurg 91:109-115, 1989.

6. HO JC, WU CY, CARRON MJ, MAUGHAN KP, SCHMIDT FJ: Titanium cerebral aneurysm clips: Characterization and performance in magnetic resonance imaging and computed tomography. J Test Eval 24:85-90, 1997.

7. KANAL E, SHELLOCK FG, LEWIN JS: Aneurysm clip testing for ferromagnetic properties: Clip variability issues. Radiology 200:576-578, 1996.

8. KEAN DM, WORTHINGTON BS, FIRTH JL, HAWKES $\mathrm{RC}$ : The effects of magnetic resonance imaging on different types of microsurgical clips. J Neurol Neurosurg Psychiatry 48:286-28, 1985 (letter).

9. KLUCZNIK RP, CARRIER DA, PYKA R, HAID RW: Placement of a ferromagnetic intracerebral aneurysm clip in a magnetic field with a fatal outcome. Radiology 187:855-856, 1993.

10. LAWTON MT, HEISERMAN JE, PRENDERGAST VC, ZABRAMSKI JM, SPETZLER RF: Titanium aneurysm Clips: Part III - Clinical application in 16 patients with subarachnoid hemorrhage. Neurosurgery 38:1170-1175, 1996.

11. LAWTON MT, HO JC, BICHARD WD, COONS JC, ZABRAMSKI JM, SPETZLER RF: Titanium aneurysm clips: Part I - Mechanical, radiological, and biocompatibility testing. Neurosurgery 38:1158-1164, 1996.

12. NEW PF, ROSEN BR, BRADY TJ, BUONANNO FS, KISTLER JP, BURT CT, HINSHAW WS, NEWHOUSE $\mathrm{JH}$, POHOST GM, TAVERAS JM: Potential hazards and artifacts of ferromagnetic and nonferromagnetic surgical and dental materials and devices in nuclear magnetic resonance imaging. Radiology 147:139-148, 1983.

13. RUPP R, EBRAHEIM NA, SAVOLAINE ER, JACKSON WT: Magnetic resonance imaging evaluation of the spine with metal implants: general safety and superior imaging with titanium. Spine 18:379-385, 1993.

14. SHELLOCK FG, CRUES JV: High-field-strength MR imaging and metallic biomedical implants: an ex vivo evaluation of deflection forces. AJR 151:389-392, 1988.

15. SHELLOCK FG, MORISOLI S, KANAL E: MR procedures and biomedical implants, materials, and devices: 1993 update. Radiology 189:587-599, 1993.

16. TOMINAGA T, SHIMIZU H, KOSHU K, KAYAMA T, YOSHIMOTO T: Magnetic resonance imaging of titanium anterior cervical spine plating systems. Neurosurgery 36:951-955, 1995.

17. WILLIAMS KD, DRAYER BP: Magnets, metal, and medical devices: The good, the bad, and the ugly. BNI Quart 5:46-52, 1989.

Original recebido em dezembro de 1998

Aceito para publicação em janeiro de 1999

\section{Endereço para correspondência:}

Mario G. Siqueira

Rua Virgílio de Carvalho Pinto, 381/apto 42

CEP 05415-030 - São Paulo, SP 